\title{
A STOKES FLOW MODEL FOR THE DRAG ON A BLOOD CELL*
}

BY

\author{
A. M. J. DAVIS (University of Alabama) \\ AND \\ K. B. RANGER (University of Toronto)
}

\begin{abstract}
The geometrical inverse of a prolate ellipsoid of revolution with respect to its center is a biconcave lens for certain values of the parameters. This is used as a model for the normal shape of a red blood cell. The Stokes flow past this body is found explicitly and an expression given for the drag.

1. Introduction. Blood is essentially a suspension of particles in an aqueous solution of variable constituents. The aqueous solution, called plasma, serves primarily as a transport vehicle for the formed elements which occupy $45-50 \%$ of the total volume. The three most important cells in the human blood are the red blood cells (R.B.C. or erythrocytes), the white blood cells (W.B.C. or leukocytes), and the blood platelets. Red blood cells are by far the most numerous and completely dominate the mechanical properties. These cells are flexible, biconcave circular disks of diameter $8 \mu \mathrm{m}$ (in most mammals) and thickness $1 \mu \mathrm{m}$ at the center and $2-3 \mu \mathrm{m}$ at the edge. There are about 5 million red cells per cubic millimeter of whole blood. A sketch of the normal shape of a red blood cell has been given by Canham and Burton [1]. For more details of the physiology of red cells the reader is referred to Pedley [2] and Dinnar [3].

In this paper a Stokes flow model is presented to determine the axisymmetric stream function for streaming flow past a single red blood cell. The geometry of the normal shape of the cell is modelled by the geometrical inverse of a prolate ellipsoid of revolution with respect to its center. In general this shape is a smooth axisymmetric biconcave lens and the stream function is found in the form of an infinite series containing Legendre functions of the first and second kind. An expression is found for the drag on the cell and there is a numerical discussion for certain values of the parameters.
\end{abstract}

\footnotetext{
*Received March 19, 1986.
} 
2. Inversion. The prolate ellipsoid given in terms of cylindrical polar coordinates $(\rho, \theta, z)$ by

$$
\frac{z^{2}}{s_{0}^{2}}+\frac{\rho^{2}}{s_{0}^{2}-1}=c^{2} \quad\left(s_{0}>1\right)
$$

is the surface $s=s_{0}$ of the elliptical coordinate system defined, for $s \geqslant 1$ and $|t| \leqslant 1$, by

$$
z=c s t, \quad \rho=c\left(s^{2}-1\right)^{1 / 2}\left(1-t^{2}\right)^{1 / 2} .
$$

This ellipsoid becomes a sphere of radius $a^{-1}$ in the limit $s_{0} \rightarrow \infty, c s_{0} \rightarrow a^{-1}(c \rightarrow 0)$ and a circular cylinder of radius $a^{-1}$ in the limit $s_{0} \rightarrow 1, c \sqrt{s_{0}^{2}-1} \rightarrow a^{-1}(c \rightarrow \infty)$.

In terms of the inverse coordinates given by

$$
z^{\prime}=\frac{z}{\rho^{2}+z^{2}}, \quad \rho^{\prime}=\frac{\rho}{\rho^{2}+z^{2}},
$$

the ellipsoid defined by (2.1) has equation

$$
\frac{z^{\prime 2}}{s_{0}^{2}}+\frac{\rho^{\prime 2}}{s_{0}^{2}-1}=c^{2}\left(\rho^{\prime 2}+z^{\prime 2}\right)^{2} .
$$

Thus, in $\left(\rho^{\prime}, \theta, z^{\prime}\right)$ space, the inverse body is evidently oblate since its axes are $\left(c s_{0}\right)^{-1}$ and $\left(c \sqrt{s_{0}^{2}-1}\right)^{-1}$ in the $z^{\prime}$ and $\rho^{\prime}$ directions respectively, i.e., the inverses of those of the ellipsoid. In particular, by letting $s_{0} \rightarrow \infty, c \rightarrow 0$ or, $s_{0} \rightarrow 1, c \rightarrow \infty$ as described above, it is readily seen that the inverse of a sphere is a sphere of inverse radius and the inverse of a circular cylinder of radius $a^{-1}$ is a closed torus of radius $\frac{1}{2} a$ whose equation is $\rho^{\prime} a=\rho^{\prime 2}+z^{\prime 2}$. The approximate form of (2.4) for small $\rho^{\prime}$ is

$$
z^{\prime 2}=\frac{1}{c^{2} s_{0}^{2}}+\left(\frac{1}{s_{0}^{2}-1}-1\right) \rho^{\prime 2}
$$

so the inverse body is concave with respect to the $z^{\prime}$-axis whenever $1 \leqslant s_{0}<\sqrt{2}$. Its surface area $S$ is given by

$$
\begin{aligned}
S & =\int_{-1}^{1} 2 \pi \rho^{\prime} \sqrt{\left(\frac{d z^{\prime}}{d t}\right)^{2}+\left(\frac{d \rho^{\prime}}{d t}\right)^{2}} d t \\
& =\frac{4 \pi}{c^{2}}\left(s_{0}^{2}-1\right)^{1 / 2} \int_{0}^{1} \frac{\left(s_{0}^{2}-t^{2}\right)^{1 / 2}}{\left(s_{0}^{2}+t^{2}-1\right)^{2}} d t
\end{aligned}
$$

after substitution of (2.2) and (2.3). Then, on setting

$$
t=s_{0}\left(s_{0}^{2}-1\right)^{1 / 2}\left[s_{0}^{2}-1+\left(2 s_{0}^{2}-1\right) y^{2}\right]^{-1 / 2},
$$

it follows that

$$
S=\frac{2 \pi}{c^{2}\left(s_{0}^{2}-1\right)}\left[\frac{s_{0}^{2}-1}{s_{0}^{2}}+\frac{s_{0}^{2}}{\left(2 s_{0}^{2}-1\right)^{1 / 2}} \tan ^{-1}\left\{\frac{\left(2 s_{0}^{2}-1\right)^{1 / 2}}{s_{0}^{2}-1}\right\}\right] .
$$

The known values $4 \pi a^{2}$ and $\pi^{2} a^{2}$, for the sphere and closed torus respectively, are readily recovered from this formula. 
Consider now the Stokes stream function $\psi(\rho, z)$ which satisfies the differential equation

$$
\left(\frac{\partial^{2}}{\partial \rho^{2}}-\frac{1}{\rho} \frac{\partial}{\partial \rho}+\frac{\partial^{2}}{\partial z^{2}}\right)^{2} \psi=0
$$

The introduction of the transformation (2.3) then shows that $\psi / r^{3}$, where $r^{2}=\rho^{2}+z^{2}$, satisfies a similar equation in $\left(\rho^{\prime}, z^{\prime}\right)$ space, i.e.,

$$
\left[\rho^{\prime} \frac{\partial}{\partial \rho^{\prime}}\left(\frac{1}{\rho^{\prime}} \frac{\partial}{\partial \rho^{\prime}}\right)+\frac{\partial^{2}}{\partial z^{\prime 2}}\right]^{2}\left(\frac{\psi}{r^{3}}\right)=0
$$

(see Ranger [4]). In particular, if

$$
\psi=\frac{1}{2} \rho^{2}\left[\frac{1}{r}-\chi\right]
$$

where $\chi$ is a function of $\rho$ and $z$ which is regular at $r=0$, then, from (2.3) with $r^{\prime}=r^{-1}$,

$$
\frac{\psi}{r^{3}}=\frac{1}{2} \rho^{\prime 2}\left[1-\frac{1}{r^{\prime}} \chi\right] \text {. }
$$

Moreover, on recalling the well-known forms of the stream function for a uniform flow or point force (Stokeslet) and noting that the boundary conditions are invariant in form, it is seen that a uniform stream $U \hat{z}^{\prime}$ disturbed by the oblate body defined by (2.4) is the inverse of the flow due to a point force of strength $4 \pi \mu U \hat{z}$ applied at the origin inside the prolate ellipsoid given by (2.1).

The drag force on the oblate body is $8 \pi \mu U$ times the coefficient of $-\rho^{\prime 2} / r^{\prime}$ in $(2.8)$ in the limit $r^{\prime} \rightarrow \infty$, i.e., $4 \pi \mu U \lim _{r \rightarrow 0} \chi$. A suitable length scale is $(S / 4 \pi)^{1 / 2}$, where $S$ is given by (2.5), and thus a nondimensional drag coefficient $C_{D}$ may be defined by

$$
C_{D}=\frac{\text { Drag }}{6 \pi \mu U(S / 4 \pi)^{1 / 2}}=\frac{4}{3}\left(\frac{\pi}{S}\right)^{1 / 2} \lim _{r \rightarrow 0} \chi .
$$

This has been scaled so as to reduce to the usual result $C_{D}=1$ for the sphere. Since the drag on a closed torus of radius $\frac{1}{2} a$ is $35.2 \mu U\left(\frac{1}{2} a\right)$ (Dorrepaal, Majumdar, O'Neill, and Ranger [5]), the corresponding drag coefficient is, according to (2.9),

$$
C_{D}=\frac{35.2}{6 \pi^{3 / 2}} \simeq 1.0536 .
$$

A formula for $C_{D}\left(s_{0}\right)$, valid for bodies in the family defined by (2.4), will be derived in the next section.

3. Solution for $\chi$. According to (2.7), $\rho^{2} \chi$ is required to satisfy (2.6) and hence

$$
\chi=\chi^{(1)}+\chi^{(3)}
$$

where

$$
\left(\frac{\partial^{2}}{\partial \rho^{2}}+\frac{k}{\rho} \frac{\partial}{\partial \rho}+\frac{\partial^{2}}{\partial z^{2}}\right) \chi^{(k)}=0 \quad(k=1,3) .
$$


By seeking separated solutions in terms of the coordinates $(s, t)$ defined by $(2.2)$, it readily follows that, in the interior of the prolate ellipsoid $\left(1 \leqslant s \leqslant s_{0},|t| \leqslant 1\right)$,

$$
\chi^{(1)}=\frac{1}{c} \sum_{n=0}^{\infty} A_{n} P_{2 n}(s) P_{2 n}(t), \quad \chi^{(3)}=\frac{1}{c} \sum_{n=1}^{\infty} B_{n} P_{2 n+1}^{\prime}(s) P_{2 n+1}^{\prime}(t)
$$

where $P_{m}$ denotes a Legendre polynomial and only even functions of $t$ have been included because $\psi$ and hence $\chi$ are evidently even in $z$. Since the origin is given by $s=1, t=0$, substitution of (3.1) and (3.2) into (2.9) yields

$$
C_{D}=\frac{2}{3}\left(\frac{4 \pi}{c^{2} s}\right)^{1 / 2}\left\{A_{0}+\sum_{n=1}^{\infty} P_{2 n}(0)\left[A_{n}+(2 n+1)^{2}(n+1) B_{n}\right]\right\}
$$

where $S$ is given by (2.5).

The coefficients $\left\{A_{n}, B_{n}\right\}$ are determined by applying the no slip conditions $\psi=0=$ $\partial \psi / \partial s$ at $s=s_{0}$. Now, in (2.7),

$$
\frac{1}{r}=\frac{1}{c}\left(s^{2}+t^{2}-1\right)^{-1 / 2}=\frac{1}{c} \sum_{n=0}^{\infty}(4 n+1) P_{2 n}(0) Q_{2 n}(s) P_{2 n}(t)
$$

according to the result (A2), where $Q_{m}$ denotes a Legendre function of the second kind. Also

$$
(4 n+1) P_{2 n}(t)=P_{2 n+1}^{\prime}(t)-P_{2 n-1}^{\prime}(t) \quad(n \geqslant 1)
$$

is a well-known recurrence relation which shows that, when (3.2) and (3.4) are substituted into (3.1) and (2.7), the equations determining the coefficients are

$$
\left.\begin{array}{c}
A_{0}-\frac{A_{1}}{5} P_{2}=Q_{0}+\frac{1}{2} Q_{2}, \quad-\frac{A_{1}}{5} P_{2}^{\prime}=Q_{0}^{\prime}+\frac{1}{2} Q_{2}^{\prime}, \\
\frac{A_{n}}{4 n+1} P_{2 n}-\frac{A_{n+1}}{4 n+5} P_{2 n+2}+B_{n} P_{2 n+1}^{\prime}=P_{2 n}(0) Q_{2 n}-P_{2 n+2}(0) Q_{2 n+2} \\
\frac{A_{n}}{4 n+1} P_{2 n}^{\prime}-\frac{A_{n+1}}{4 n+5} P_{2 n+2}^{\prime}+B_{n} P_{2 n+1}^{\prime \prime}=P_{2 n}(0) Q_{2 n}^{\prime}-P_{2 n+2}(0) Q_{2 n+2}^{\prime}
\end{array}\right\} \quad(n \geqslant 1)
$$

where the Legendre functions are here and below to be evaluated at $s=s_{0}$ unless otherwise stated. Then, on writing

$$
\alpha_{n}=\frac{A_{n}}{4 n+1} P_{2 n}-P_{2 n}(0) Q_{2 n} \quad(n \geqslant 0)
$$

and using the Wronskian $Q_{m} P_{m}^{\prime}-Q_{m}^{\prime} P_{m}=\left(s_{0}^{2}-1\right)^{-1}(m \geqslant 0)$, the above equations have the simpler form

$$
\begin{gathered}
\alpha_{0}=\alpha_{1}=\frac{s_{0}}{2\left(s_{0}^{2}-1\right)}, \\
\alpha_{n}-\alpha_{n+1}+B_{n} P_{2 n+1}^{\prime}=0, \\
\left.\alpha_{n} \frac{P_{2 n}^{\prime}}{P_{2 n}}-\alpha_{n+1} \frac{P_{2 n+2}^{\prime}}{P_{2 n+2}}+B_{n} P_{2 n+1}^{\prime \prime}=\frac{1}{s_{0}^{2}-1}\left[-\frac{P_{2 n}(0)}{P_{2 n}}+\frac{P_{2 n+2}(0)}{P_{2 n+2}}\right]\right\} \quad(n \geqslant 1) .
\end{gathered}
$$


Next, define

$$
\gamma_{n}=P_{2 n} P_{2 n+1}^{\prime \prime}-P_{2 n}^{\prime} P_{2 n+1}^{\prime}=P_{2 n} P_{2 n-1}^{\prime \prime}-P_{2 n}^{\prime} P_{2 n-1}^{\prime} \quad(n \geqslant 1),
$$

the two expressions being equal because of (3.5). Then the elimination of $B_{n}$ for each $n \geqslant 1$ yields a set of difference equations whose solution is evidently

$$
\alpha_{n}=\frac{P_{2 n}(0) s_{0} P_{2 n}^{\prime}}{\gamma_{n}\left(s_{0}^{2}-1\right)} \quad(n \geqslant 1)
$$

after using the recurrence relations

$$
\begin{array}{cl}
P_{m+1}^{\prime}=(m+1) P_{m}+s_{0} P_{m}^{\prime} & (m \geqslant 0), \\
P_{m-1}^{\prime}=-m P_{m}+s_{0} P_{m}^{\prime} & (m \geqslant 1), \\
(2 n+1) P_{2 n}(0)+(2 n+2) P_{2 n+2}(0)=0 & (n \geqslant 0) .
\end{array}
$$

Hence, by substituting (3.7) and (3.9) into (3.6) and the equation $B_{n} P_{2 n+1}^{\prime}=\alpha_{n+1}-\alpha_{n}$ $(n \geqslant 1)$, the summation in the drag coefficient formula (3.3) can be written, after some further manipulation,

$$
\begin{aligned}
& A_{0}+\sum_{n=1}^{\infty} P_{2 n}(0)\left[A_{n}+(2 n+1)^{2}(n+1) B_{n}\right]=\sum_{n=0}^{\infty}(4 n+1)\left[P_{2 n}(0)\right]^{2} \frac{Q_{2 n}}{P_{2 n}} \\
& +\frac{s_{0}}{s_{0}^{2}-1} \sum_{n=1}^{\infty} \frac{\left[P_{2 n}(0)\right]^{2}}{\gamma_{n}} P_{2 n}^{\prime}\left\{\frac{4 n+1}{P_{2 n}}-\frac{2 n^{2}(2 n-1)}{P_{2 n-1}^{\prime}}-\frac{(2 n+1)^{2}(n+1)}{P_{2 n+1}^{\prime}}\right\} .
\end{aligned}
$$

$C_{D}\left(s_{0}\right)$ is then obtained by substituting (2.5) and (3.10) into (3.3) and would appear to increase from unity to 1.0536 as $s_{0}$ decreases from infinity to unity.

4. Application to red blood cell. The lengths of the axes of the oblate body can be matched with those of a red blood cell by setting

$$
\left(c s_{0}\right)^{-1}=1 \mu m, \quad\left(c \sqrt{s_{0}^{2}-1}\right)^{-1}=4 \mu m
$$

whence

$$
c^{-1}=\frac{4}{\sqrt{15}} \mu m, \quad s_{0}=\frac{4}{\sqrt{15}} \simeq 1.0328 .
$$

This might suggest that the bounding membrane of given surface area is shaped so as to achieve as large a value of $C_{D}$ as possible, i.e., a $5 \%$ gain over the sphere, without approaching the unacceptable curvatures of the cusp-like behaviour. However, when the above numerical values are substituted into (2.5) and the corresponding formula

$$
V=\frac{\pi s_{0}}{2 c^{3}\left(s_{0}^{2}-1\right)^{3 / 2}}\left\{\frac{\pi}{2}+\left(s_{0}^{2}-1\right)^{1 / 2}-\tan ^{-1}\left(s_{0}^{2}-1\right)^{1 / 2}-\left(s_{0}^{2}-1\right)^{3 / 2}\left(\frac{1}{s_{0}^{2}}-\frac{2}{3 s_{0}^{4}}\right)\right\}
$$

for the volume $V$, the values obtained are $S=157.9(\mu \mathrm{m})^{2}$ and $V=163.1(\mu \mathrm{m})^{3}$ whereas a blood cell has surface area $135(\mu \mathrm{m})^{2}$ and volume $94(\mu \mathrm{m})^{3}$. Clearly the mathematically convenient family of surfaces defined by (2.4) does not allow sufficient reduction of 
enclosed volume in relation to surface area. The required axial lengths are achieved by undue "pinching" at the minor axis towards the shape of the closed torus but the dimensionless comparison of volume and surface area, defined by

$$
\text { sphericity index }=s_{p}=\left(6 \pi^{1 / 2} V\right)^{2 / 3} / S,
$$

is, for the surfaces considered, confined to the range $91.4 \%$ to unity. For the blood cell its value is $74.0 \%$.

The mathematical analysis has three other limitations in the context of blood flow. The red blood cells fill $42-46 \%$ of the volume occupied by the blood and hence should not be considered in isolation. They are generally observed to move in a shear flow rather than a uniform stream. They are not solid but are bounded by an elastic membrane which resists deformation but allows relative motion of the interior hemoglobin.

Nevertheless the mathematics provides a useful pointer towards a significant property. If the drag coefficient is instead based on a length scale determined by volume then comparison of (2.9) and (4.1) shows that $C_{D}$ must be modified by the factor $s_{p}^{-1 / 2}$ which for the red blood cell is 1.1625 . Thus it may be reasonably suggested that for a given volume of hemoglobin, the bounding membrane has evolved in a way that produces a gain in drag of the order of $20 \%$ compared with the spherical shape and hence a consequent increase in the efficiency with which the cells are transported in the blood flow.

Appendix. The expansion of $\left(s^{2}+t^{2}-1\right)^{-1 / 2}(|t| \leqslant 1, s>1)$ in terms of $\left\{P_{2 n}(t)\right.$ : $n \geqslant 0\}$ does not appear to be listed in standard references but can be obtained by evaluating

$$
\begin{aligned}
I_{n}(s) & =\int_{-1}^{1} \frac{P_{2 n}(t) d t}{\left(s^{2}+t^{2}-1\right)^{1 / 2}} \\
& =\sum_{h=0}^{\infty} \frac{\Gamma\left(m+n+\frac{1}{2}\right)}{s^{2 m+2 n+1} \Gamma\left(\frac{1}{2}\right) \Gamma(m+n+1)} \int_{-1}^{1} P_{2 n}(t)\left(1-t^{2}\right)^{m+n} d t
\end{aligned}
$$

where $0<1-t^{2} \leqslant 1<s^{2}$ ensures that the expansion is valid and only the $n$th and higher powers of $\left(1-t^{2}\right)$ can contribute. The corrected form

$$
\int_{-1}^{1}\left(1-x^{2}\right)^{\lambda-1} P_{\nu}(x) d x=\frac{(\Gamma(\lambda))^{2} \Gamma\left(\frac{1}{2} \nu+\frac{1}{2}\right) \cos \frac{1}{2} \pi \nu}{\Gamma\left(\lambda+\frac{1}{2} \nu+\frac{1}{2}\right) \Gamma\left(\lambda-\frac{1}{2} \nu\right) \Gamma\left(\frac{1}{2} \nu+1\right)}
$$

of equation (1) in section 7.132 of Gradshteyn and Ryzhik [6] together with the definition

$$
Q_{2 n}(s)=\frac{\Gamma(2 n+1) \Gamma\left(\frac{1}{2}\right)}{2^{2 n+1} \Gamma\left(2 n+\frac{3}{2}\right) s^{2 n+1}} F\left(n+1, n+\frac{1}{2} ; 2 n+\frac{3}{2} ; \frac{1}{s^{2}}\right),
$$

given in section 8.703 of the same reference, can then be used with the series expansion of the hypergeometric function to show that

$$
I_{n}(s)=2 \frac{(-1)^{n} \Gamma\left(n+\frac{1}{2}\right)}{\Gamma(n+1) \Gamma\left(\frac{1}{2}\right)} Q_{2 n}(s)=2 P_{2 n}(0) Q_{2 n}(s) .
$$


Hence, from the definition (A1),

$$
\left(s^{2}+t^{2}-1\right)^{-1 / 2}=\sum_{n=0}^{\infty}(4 n+1) P_{2 n}(0) Q_{2 n}(s) P_{2 n}(t) \text {. }
$$

\section{REFERENCES}

[1] P. B. Canham and A. C. Burton, Circulation research 22, 405 (1968)

[2] T. J. Pedley, The fluid mechanics of large blood vessels, Cambridge University Press (1980), pp. 29-31

[3] V. Dinnar, Cardiovascular fluid dynamics, Boca Raton, Florida: CRC Press (1981), pp. 23-28

[4] K. B. Ranger, A Stokes flow treated by the method of inversion, Quart. J. Mech. \& Appl. Math. 18, 277-285 (1965)

[5] J. M. Dorrepaal, S. R. Majumdar, M. E. O’Neill, and K. B. Ranger, A closed torus in Stokes flow, Quart. J. Mech. \& Appl. Math. 29, 381-387 (1976)

[6] I. S. Gradshteyn and I. M. Ryzhik, Tables of integrals, series, and products (enlarged edition), ed. A. Jeffrey, New York: Academic Press (1980) 